\title{
LncRNA-UCA1 enhances MMP-13 expression by inhibiting miR- 204-5p in human chondrocytes
}

\author{
Guodong Wang ${ }^{1, *}$, Xianmin Bu${ }^{2, *}$, Yuanmin Zhang ${ }^{1, *}$, Xiaowei Zhao ${ }^{1}$, Ying Kong ${ }^{1}$, \\ Longfei Ma ${ }^{1}$, Shuaishuai Niu ${ }^{1}$, Bin Wu ${ }^{1}$ and Chunyang Meng ${ }^{1}$ \\ ${ }^{1}$ Department of Orthopaedics, Affiliated Hospital of Jining Medical University, Jining, Shandong, China \\ ${ }^{2}$ Department of Pathology, Shandong Jining No.1 People's Hospital, Jining, Shandong, China \\ *These authors contributed equally to this work
}

Correspondence to: Chunyang Meng, email: frvr11@yeah.net

Keywords: osteoarthritis, long noncoding RNAs, UCA1, miR-204-5p, MMP-13

Received: June 02, 2017 Accepted: July 25, $2017 \quad$ Published: August 10, 2017

Copyright: Wang et al. This is an open-access article distributed under the terms of the Creative Commons Attribution License 3.0 (CC BY 3.0), which permits unrestricted use, distribution, and reproduction in any medium, provided the original author and source are credited.

\section{ABSTRACT}

Osteoarthritis (OA) is a common degenerative disease characterized by degeneration of articular cartilage. Increasing studies showed that long noncoding RNAs (IncRNAs) play important roles in the cartilage damage. However, little is known about the role of UCA1 in the osteoarthritis. The expression level of UCA1 was upregulated in the OA cartilage. Overexpression of UCA1 suppressed the miR-204-5p expression in the chondrocytes. The expression of miR-204-5p was downregulated in the OA cartilage. Moreover, the expression of miR-204-5p was negatively correlated with the UCA1 expression in the OA cartilage. Elevated expression of UCA1 promoted the chondrocytes cell proliferation and overexpression of miR-204-5p suppressed chondrocytes cell proliferation. In addition, overexpression of UCA1 decreased the expression of the type II collagen and type IV collagen expression in the chondrocytes. Elevated expression of miR-204-5p promoted the type II collagen and type IV collagen expression in the chondrocytes. We idetified MMP-13 was a direct target gene of miR-204-5p in the chondrocytes. Overexpression of UCA1 enhanced the MMP-13 expression in the chondrocytes. Elevated expression of UCA1 regulated the chondrocytes cell proliferation and collagen expression through inhibiting the miR-204-5p expression.These results suggested that UCA1 played as an important regulator of survival and matrix synthesis of chondrocytes partly through suppressing the miR-204-5p expression.

\section{INTRODUCTION}

Osteoarthritis (OA) is a degenerative disease of the joints and is regarded by tenderness, pain, crepitus, limited movement, which is the most prevalent cause of mobility-associated disability [1-4]. The pathogenesis of $\mathrm{OA}$ is multifactorial and involves the interaction of several factors $[5,6]$. The etiology of OA is also complex such as failure of nutrient supply, genetic predisposition, trauma and abnormal mechanical loading [7-11].

Long noncoding RNAs (lncRNAs) are a group of the noncoding RNA family and are longer than 200 nucleotides [12-15]. Accumulating evidences demonstrated that lncRNAs are involved in several cell biological processes including cell growth, differentiation, invasion, cell cycle progression, migration and apoptosis [16-20]. Additionally, lncRNAs misexpression was found in several tumors such as retinoblastoma, gastric cancer, endometrial carcinoma, breast cancer, and colorectal cancer $[15,21-25]$. Recent studies have showed that lncRNAs play a crucial role in the development of arthritis and the joint homeostasis maintenance [26]. Su et al. [26]. showed that maternally expressed gene 3 (MEG3) was downregulated in OA patients compared to normal cartilage samples. Human urothelial carcinoma associated 1 (UCA1) was originally found to be upregulated in bladder tumor and was demonstrated to serve as an important regulator of cell proliferation, invasion and migration [27-30]. UCA1 was found to be upregulated in tongue squamous cell carcinomas, gastric cancer, breast 
cancer and bladder cancer [28, 31-34]. However, the functional role of UCA1 in OA development is still not documented.

In this study, we sought to the expression of UCA1 in the OA cartilage and normal cartilage. We showed that the expression level of UCA1 was upregulated in the OA cartilage. Overexpression of UCA1 suppressed the miR204-5p expression and enhanced the MMP-13 expression in the chondrocytes.

\section{RESULTS}

\section{The expression of UCA1 was upregulated in the OA cartilage}

We firstly determined the expression of UCA1 in the OA cartilage and normal cartilage. As shown in the Figure 1, the expression level of UCA1 was highest in the moderate and severe group compared to in the normal cartilage and mild OA cartilage. The data indicated that the expression level of UCA1 was upregulated in the OA cartilage.

\section{The expression of miR-204-5p was downregulated in the OA cartilage}

The expression of UCA1 was significantly upregulated in the chondrocytes after treated with pcDNAUCA1 (Figure 2A). Overexpression of UCA1 suppressed the miR-204-5p expression in the chondrocytes (Figure $2 \mathrm{~B})$. We then determined the expression of miR-204$5 p$ in the OA cartilage and normal cartilage. As shown in the Figure 2C, the expression level of miR-204-5p was lowest in the moderate and severe group compared to in the normal cartilage and mild OA cartilage. The data indicated that the expression level of miR-204-5p was downregulated in the OA cartilage. Moreover, the expression of miR-204-5p was negatively correlated with the UCA1 expression in the OA cartilage (Figure 2D).

\section{miR-204-5p suppressed the MMP-13 expression in the chondrocytes}

We identified a potential miR-204-5p binding sequence in the 3'UTR of MMP-13 through using TargetScanHuman miRNA target prediction software (Figure 3A). The expression of miR-204-5p was significantly upregulated in the chondrocytes after treated with miR-204-5p mimic (Figure 3B). As shown in the Figure 3C, miR-204-5p decreased the luciferase activity of MMP-13-WT plasmid, but not of MMP-13-Mut, manifesting that MMP-13 was one of miR-204-5p direct targets. Overexpression of miR-204-5p suppressed MMP13 expression in the chondrocytes (Figure 3D). Elevated expression of miR-204-5p also inhibited the protein expression of MMP-13 in the chondrocytes (Figure 3E).
Overexpression of UCA1 promoted the MMP-13 expression in the chondrocytes (Figure 3F).

\section{Elevated expression of UCA1 promoted the chondrocytes cell proliferation and miR-204-5p suppressed chondrocytes cell proliferation}

Overexpression of UCA1 increased the chondrocytes cell proliferation (Figure 4A). Elevated expression of UCA1 promoted the ki-67 expression in the chondrocytes (Figure 4B). Elevated expression of miR204-5p suppressed the chondrocytes cell proliferation (Figure 4C). Ecoptic expression of miR-204-5p inhibited the ki-67 expression in the chondrocytes (Figure 4D).

\section{Overexpression of UCA1 decreased the expression of the type II collagen and type IV collagen}

Overexpression of UCA1 suppressed the type II collagen (Figure 5A) and type IV collagen (Figure 5B) expression in the chondrocytes. In addition, elevated expression of miR-204-5p promoted the type II collagen (Figure 5C) and type IV collagen (Figure 5D) expression in the chondrocytes.

\section{Elevated expression of UCA1 regulated the chondrocytes cell proliferation and collagen expression through inhibiting the miR-204-5p expression}

We restored miR-204-5p expression in the UCA1 overexpressing-chondrocytes to study whether miR-204-5p was involved in the function of UCA1 in chondrocytes. Elevated expression of miR-204-5p inhibited chondrocytes cell proliferation, reversing UCA1-induced chondrocytes cell proliferation (Figure 6A). Overexpression of miR204-5p also inhibited the ki-67 expression in the UCA1 overexpressing-chondrocytes (Figure 6B). Moreover, elevated expression of miR-204-5p suppressed the MMP13 expression in the UCA1 overexpressing-chondrocytes (Figure 6C). Ecoptic expression of miR-204-5p enhanced the type II collagen (Figure 6D) and type IV collagen (Figure 6E) expression in the UCA1 overexpressingchondrocytes.

\section{DISCUSSION}

In our study, we found that the expression level of UCA1 was upregulated in the OA cartilage. Overexpression of UCA1 suppressed the miR-204-5p expression in the chondrocytes. The expression of miR-204-5p was downregulated in the OA cartilage. Moreover, the expression of miR-204-5p was negatively correlated with the UCA1 expression in the OA 


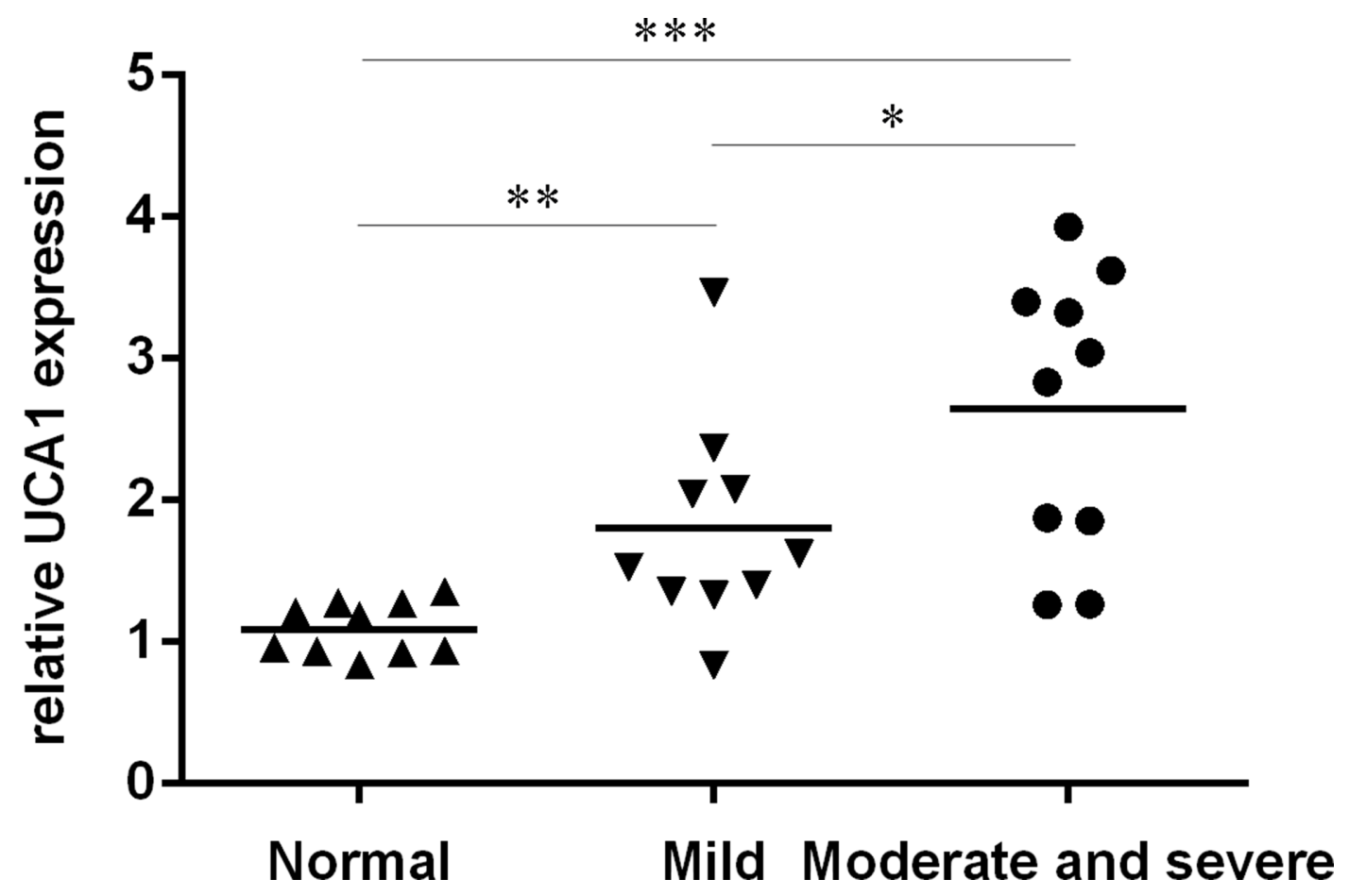

Figure 1: The expression of UCA1 was upregulated in the OA cartilage. The expression of UCA1 in the OA cartilage and normal cartilage was determined by qRT-PCR. $* p<0.05, * * p<0.01$ and $* * * p<0.001$.

A

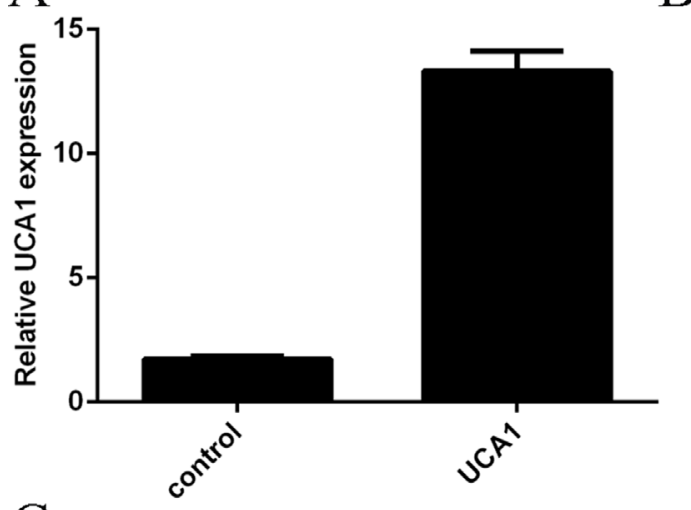

C

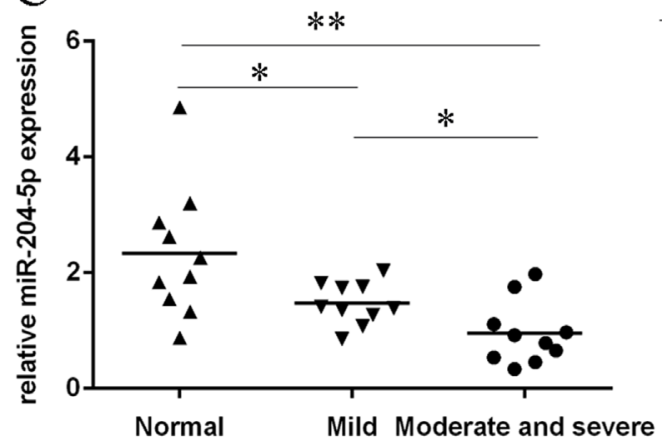

B

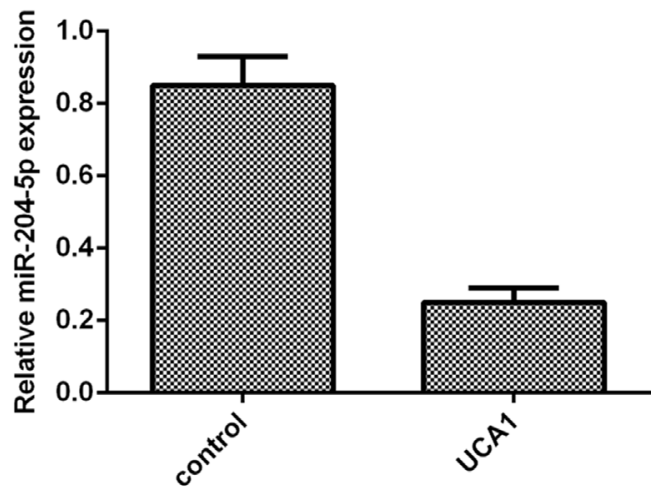

$\mathrm{D}$

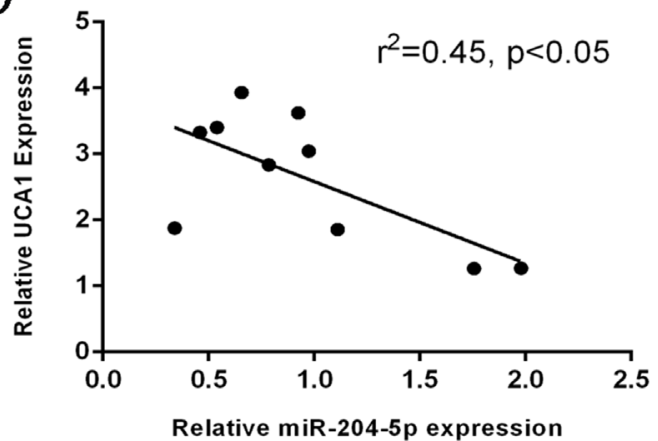

Figure 2: The expression of miR-204-5p was downregulated in the OA cartilage. (A) The expression of UCA1 was measured in the chondrocytes after treated with pcDNA-UCA1. (B) Overexpression of UCA1 suppressed the miR-204-5p expression in the chondrocytes. (C) The expression level of miR-204-5p in the normal cartilage and OA cartilage was measured by qRT-PCR. (D) The expression of miR-204-5p was negatively correlated with the UCA1 expression in the OA cartilage. ${ }^{*} p<0.05$ and ${ }^{* *} p<0.01$. 
cartilage. Elevated expression of UCA1 promoted the chondrocytes cell proliferation and overexpression of miR-204-5p suppressed chondrocytes cell proliferation. In addition, overexpression of UCA1 decreased the expression of the type II collagen and type IV collagen expression in the chondrocytes. Elevated expression of miR-204-5p promoted the type II collagen and type IV collagen expression in the chondrocytes. We identified MMP-13 was a direct target gene of miR-204-5p in the chondrocytes. Overexpression of UCA1 enhanced the MMP-13 expression in the chondrocytes. Elevated expression of UCA1 regulated the chondrocytes cell proliferation and collagen expression through inhibiting the miR-204-5p expression. These results suggested that IncRNA UCA1 played as an important regulator of survival and matrix synthesis of chondrocytes partly through suppressing the miR-204-5p expression.

Previous studies demonstrated that IncRNA UCA1 played important roles in the development of tumors such as renal cell carcinoma, gastric cancer, colon cancer, hepatocellular carcinoma and osteosarcoma [34-38]. For example, Jiao et al. [39]. demonstrated that UCA1 expression was upregulated in the esophageal cancer tissues and ecoptic expression of UCA1 increased esophageal cancer cell proliferation by regulating the miR-204 and Sox4 expression. Chen et al. [40]. showed that UCA1 expression level was upregulated in the pancreatic cancer samples and the konckdown expression

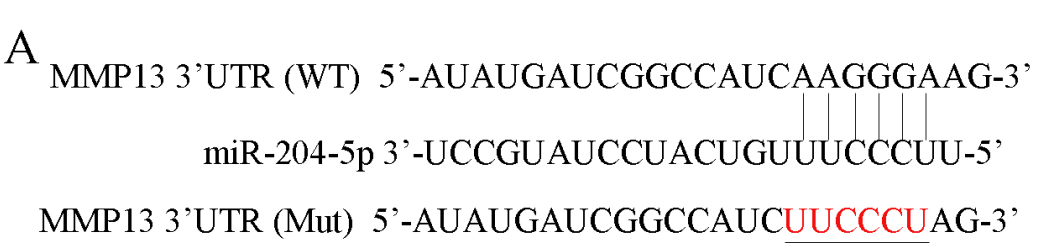

$\mathrm{C}$

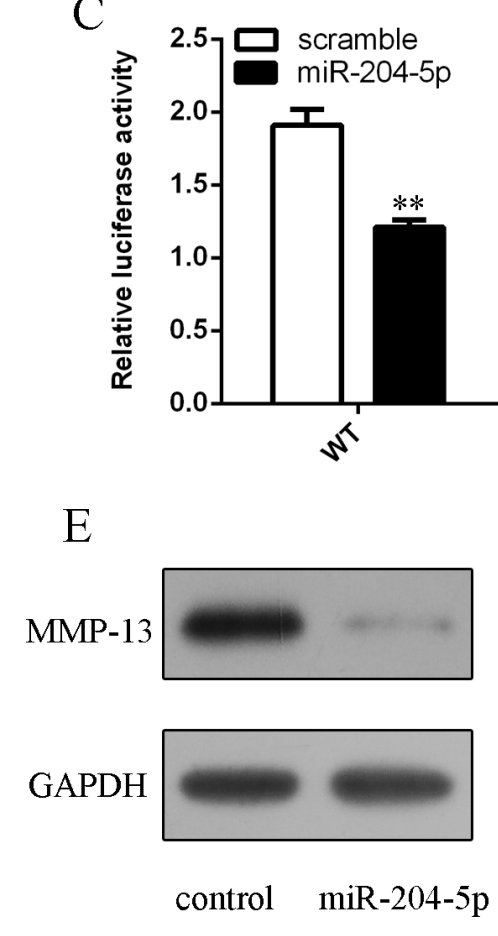

$\mathrm{F}$

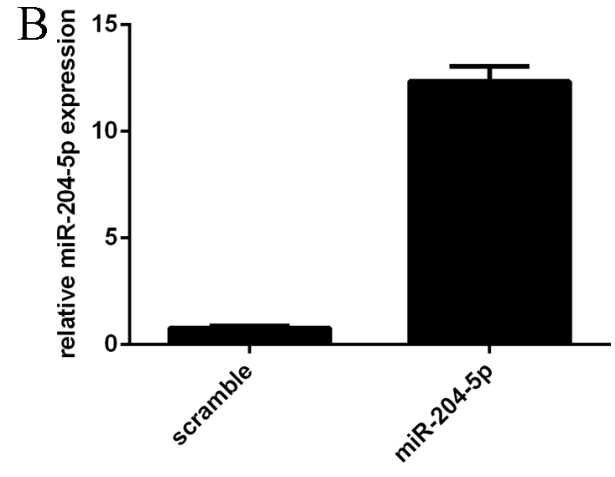

$\mathrm{D}$

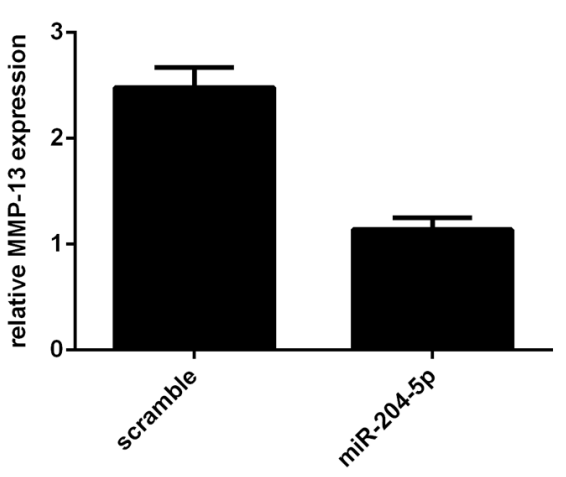

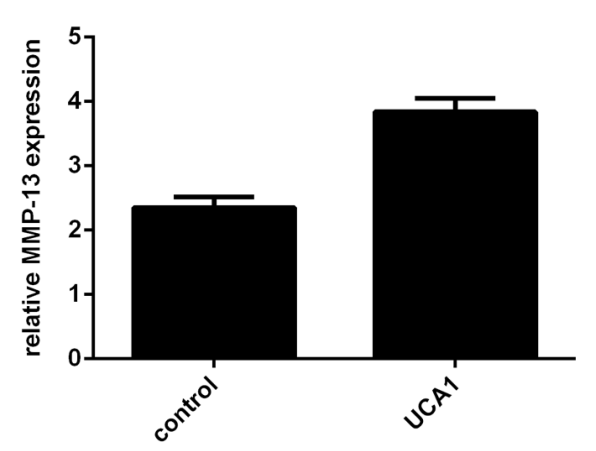

Figure 3: miR-204-5p suppressed the MMP-13 expression in the chondrocytes. (A) There is a potential miR-204-5p binding sequence in the 3'UTR of MMP-13 through by using TargetScanHuman miRNA target prediction software. (B) The expression of miR204-5p in the chondrocytes was measured by qRT-PCR. (C) miR-204-5p decreased the luciferase activity of MMP-13-WT plasmid, but not of MMP-13-Mut, manifesting that MMP-13 was one of miR-204-5p direct targets. (D) Overexpression of miR-204-5p suppressed MMP-13 expression in the chondrocytes. (E) The protein expression of MMP-13 in the chondrocytes was analyzed by western blot. (F) Overexpression of UCA1 promoted the MMP-13 expression in the chondrocytes. ${ }^{*} p<<0.01$. 
of UCA1 suppressed pancreatic cancer cell proliferation and increased the apoptotic rate and induced the cell cycle arrest. Lu et al. [41]. demonstrated that UCA1 expression level was higher in the lymph node metastasis samples than in the endometrial cancer tissues and the proliferative endometrium. Inhibition of UCA1 suppressed the endometrial cancer cell migration and invasion. However, the role of UCA1 in OA is still unknown. In this study, we found that the expression level of UCA1 was highest in the moderate and severe group compared to in the normal cartilage and mild OA cartilage. The data indicated that the expression level of UCA1 was upregulated in the OA cartilage. Elevated expression of UCA1 promoted the chondrocytes cell proliferation and overexpression of miR-204-5p suppressed chondrocytes cell proliferation. There results suggested that UCA1 play crucial roles in pathogenesis and progression of OA.

It has showed that lncRNAs act as regulators for miRNAs expression regulation. Previous studies showed that UCA1 promoted gemcitabine/cisplatin resistance by CREB regulating miR-196a-5p expression in the bladder cancer cells [28]. Fang et al. [34]. demonstrated that
UCA1 ilncreased multi-drug resistance of gastric cancer through downregulating miR-27b expression. Bian et al. showed that UCA1 enhanced the 5-fluorouracil resistance and cell proliferation in the colorectal cancer through regulating the miR-204-5p expression. In line with this, we demonstrated that overexpression of UCA1 suppressed the miR-204-5p expression in the chondrocytes. The expression of miR-204-5p was downregulated in the OA cartilage. Moreover, the expression of miR-204-5p was negatively correlated with the UCA1 expression in the OA cartilage. Elevated expression of miR-204$5 p$ promoted the type II collagen and type IV collagen expression in the chondrocytes. Moreover, we idetified MMP-13 was a direct target gene of miR-204-5p in the chondrocytes. Previous data demonstrated that MMP13 was at low expression level in the articular cartilage during physiologic ECM turnover and was upregulated in human OA. MMP-13 can degrade the expressions of type 2 collagen and aggrecan. Furthermore, we showed that elevated expression of UCA1 regulated the chondrocytes cell proliferation and collagen expression through inhibiting the miR-204-5p expression.

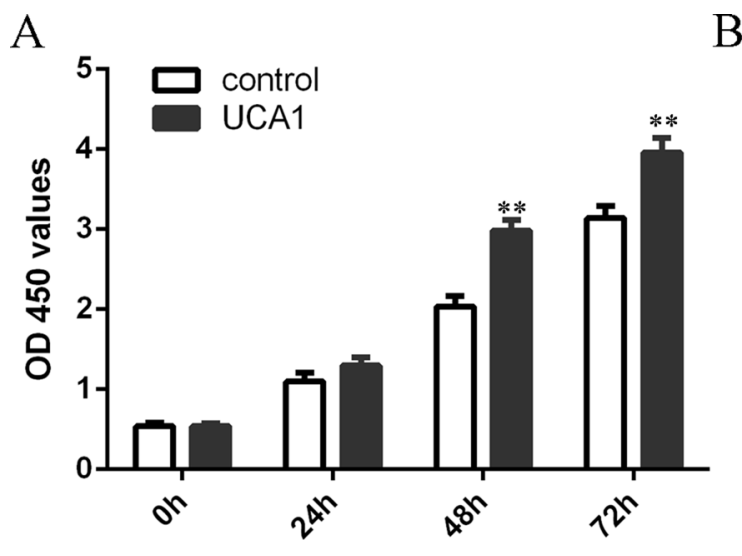

B

C

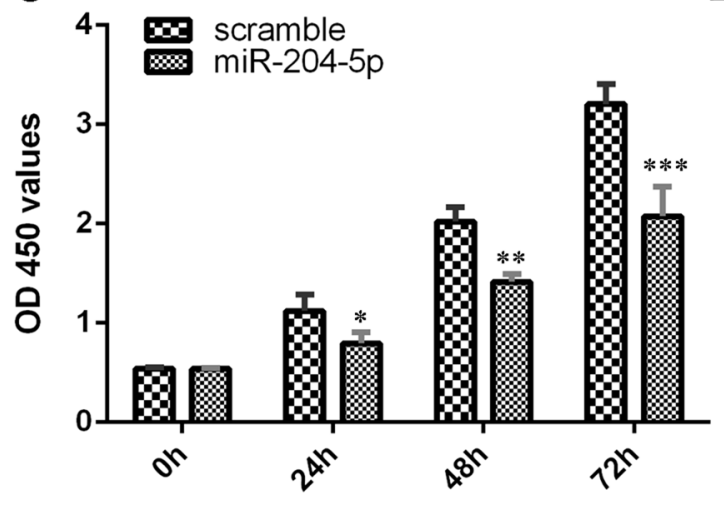

$\mathrm{D}$
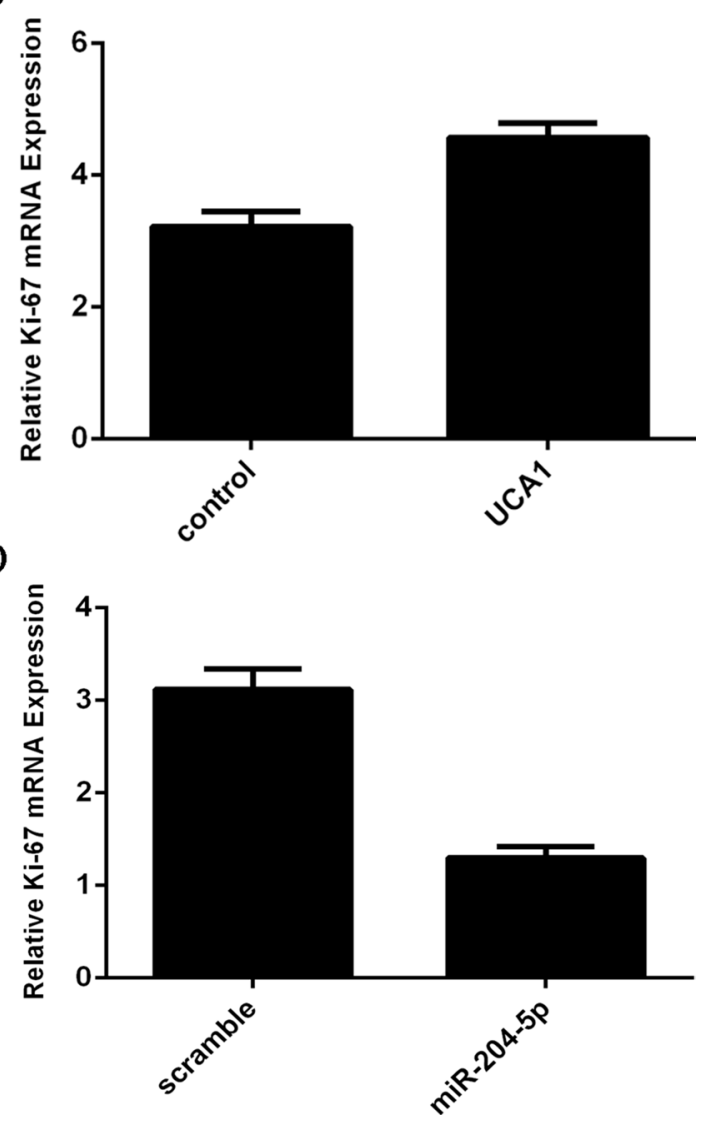

Figure 4: Elevated expression of UCA1 promoted the chondrocytes cell proliferation and miR-204-5p suppressed chondrocytes cell proliferation. Overexpression of UCA1 increased the chondrocytes cell proliferation (A). Elevated expression of UCA1 promoted the ki-67 expression in the chondrocytes (B). Elevated expression of miR-204-5p suppressed the chondrocytes cell proliferation (C). Ecoptic expression of miR-204-5p inhibited the ki-67 expression in the chondrocytes $(\mathbf{D})$. ${ }^{*} p<0.05, * * p<0.01$ and $* * * p<0.001$. 
In conclusion, we indicated that expression level of UCA1 was upregulated in the OA cartilage. Overexpression of UCA1 suppressed the miR-204-5p expression and enhanced the MMP-13 expression in the chondrocytes. These data provide the possibility of UCA1/ miR-204-5p as therapeutic targets for the treatment of OA.

\section{MATERIALS AND METHODS}

\section{Tissue samples}

Normal knee cartilage samples were collected from patitents which were underwent the amputation due to trauma with no OA or rheumatoid arthritis history. Cartilage tissues of OA were obtained from OA patients who underwent knee replacement surgery. The diagnosed of these patients were followed to the criteria of American College of Rheumatology. This study was approved by Research Ethics Committee of the affiliated hospital of jining medical university and complied with Declaration of Helsinki. The written informed consent was collected from each participant. All cartilage tissues were divided into three groups following to the Kellgren/Lawrence Criterion: normal cartilage $(\mathrm{K} / \mathrm{L}$, Grade 0$)$ for the normal group, low grade of OA cartilage (K/L Grade I and II) for the mild group, and high grade of OA cartilage $(\mathrm{K} / \mathrm{L}$, Grade III and IV) for the moderate and severe group.

\section{Cell culture and cell transfection}

The human chondrocytes cell line C28/I2 (immortalized juvenile costal chondrocytes cell line) was purchased from ATCC (American Type Culture
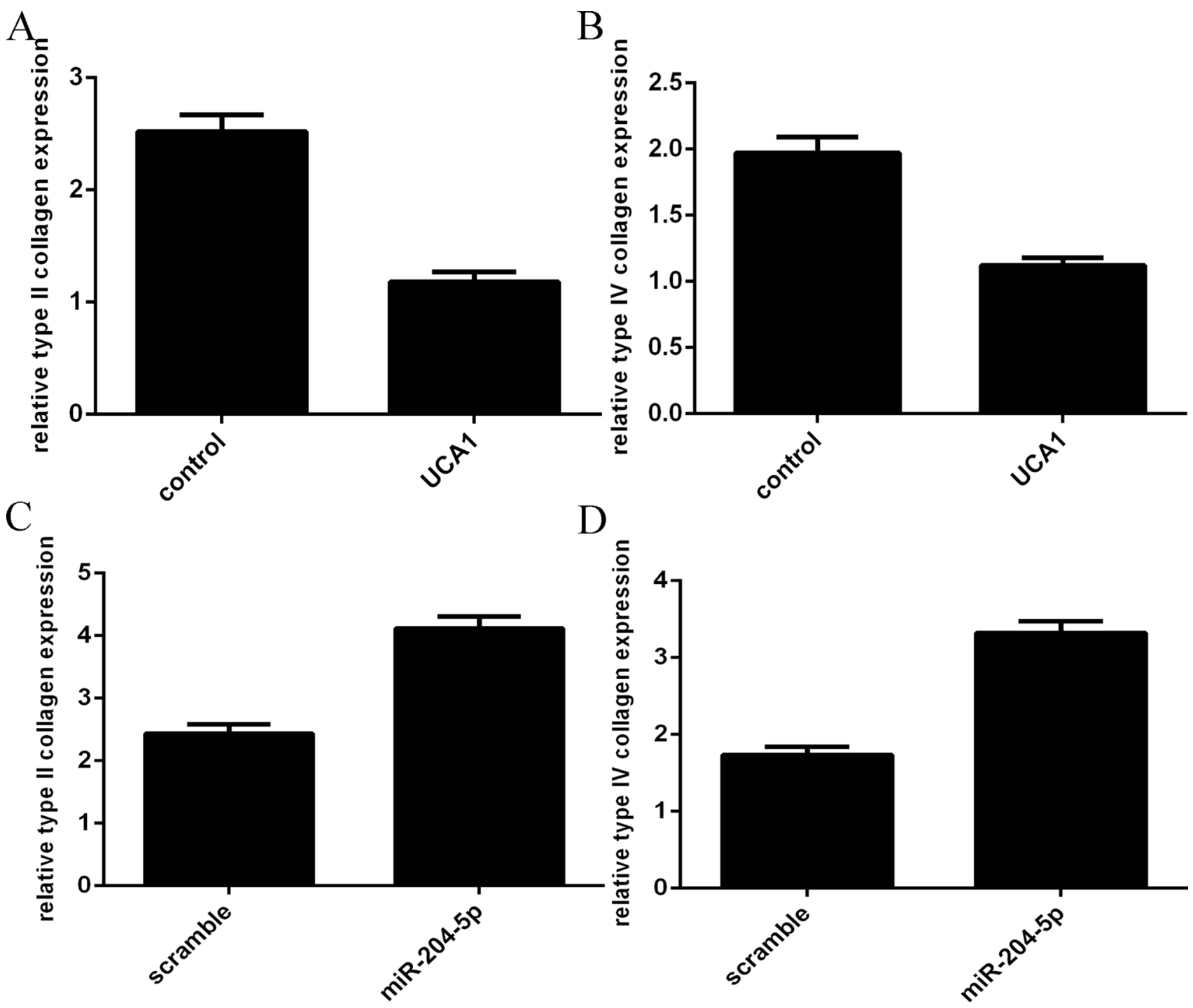

Figure 5: Overexpression of UCA1 decreased the expression of the type II collagen and type IV collagen. (A) Overexpression of UCA1 suppressed the type II collagen expression in the chondrocytes. (B) The expression of type IV collagen in the chondrocytes was measured by qRT-PCR. (C) Elevated expression of miR-204-5p promoted the type II collagen expression. (D) The expression of type IV collagen in the chondrocytes was measured by qRT-PCR. 
A

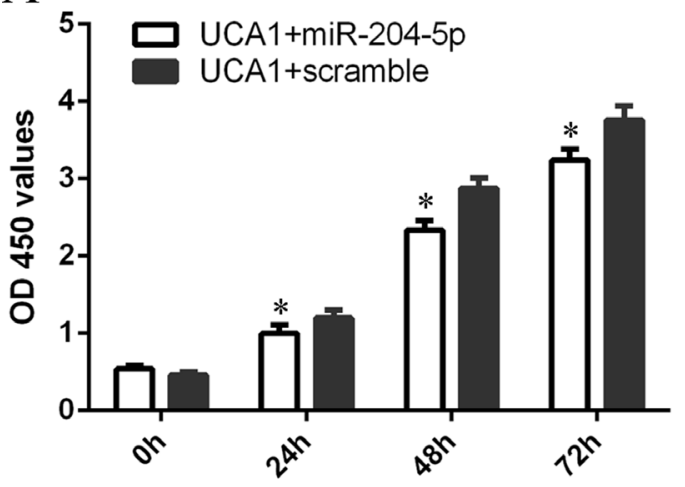

$\mathrm{C}$

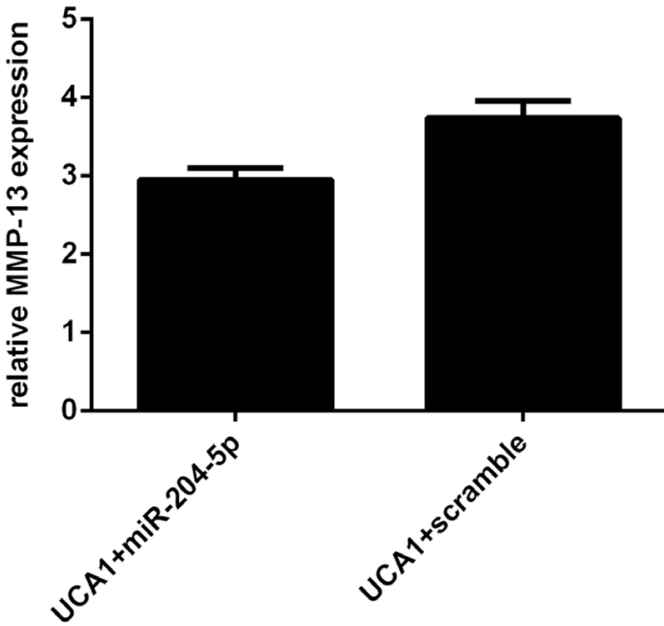

$\mathrm{B}$

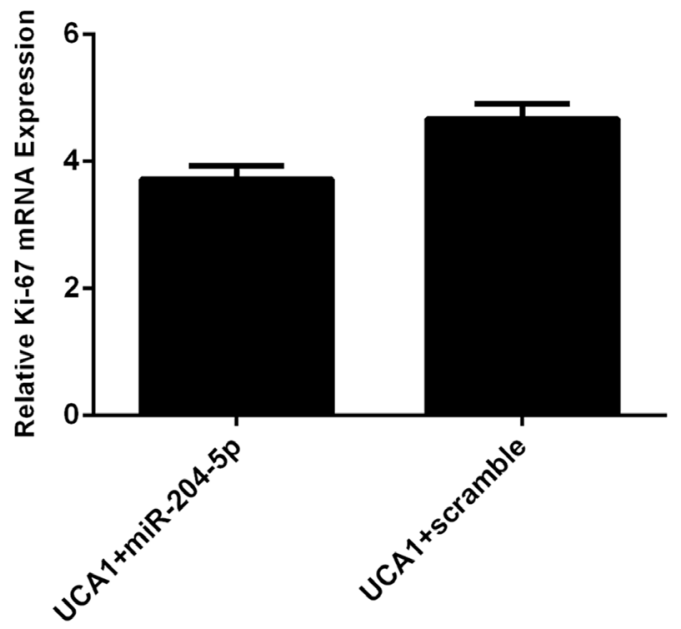

$\mathrm{D}$

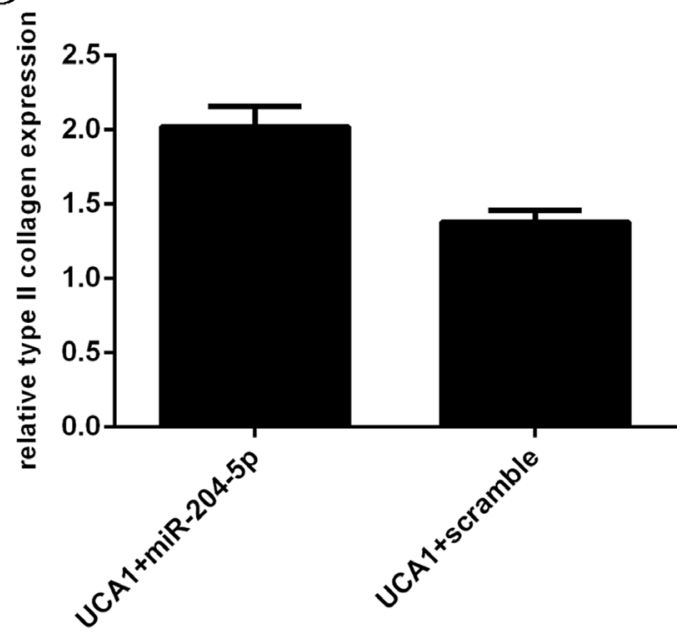

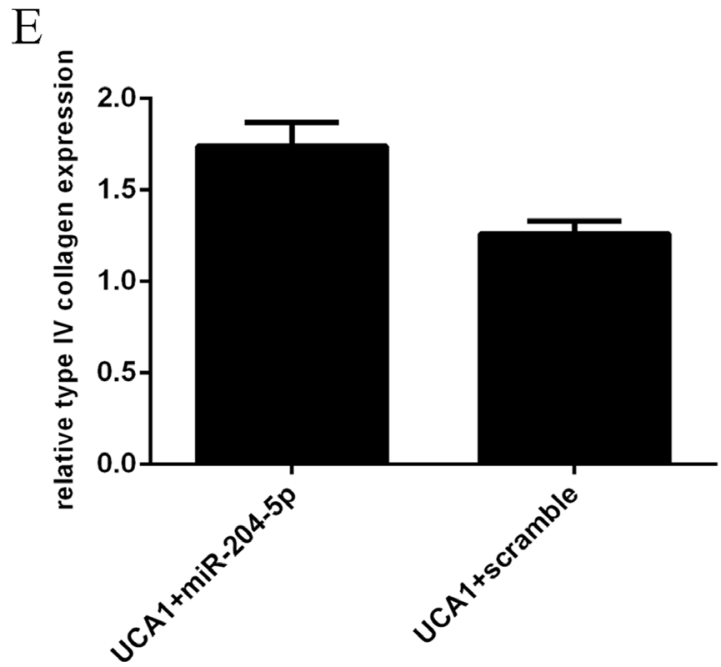

Figure 6: Elevated expression of UCA1 regulated the chondrocytes cell proliferation and collagen expression through inhibiting the miR-204-5p expression. (A) Elevated expression of miR-204-5p inhibited chondrocytes cell proliferation, reversing UCA1-induced chondrocytes cell proliferation. (B) Overexpression of miR-204-5p also inhibited the ki-67 expression in the UCA1 overexpressing-chondrocytes. (C) Elevated expression of miR-204-5p suppressed the MMP-13 expression in the UCA1 overexpressingchondrocytes. (D) Ecoptic expression of miR-204-5p enhanced the type II collagen expression in the UCA1 overexpressing-chondrocytes. (E) Ecoptic expression of miR-204-5p enhanced the type IV collagenexpression in the UCA1 overexpressing-chondrocytes. ${ }^{*} p<0.05$. 
Collection, USA) and was cultured in the DMEM with fetal bovine serum (FBS), penicillin, and streptomycin. LncRNA UCA1 and control vector, miR-204-5p mimic and scramble were purchased from GenePharma (Shanghai, China). Cell transfection was performed by using the Lipofectamine 2000 (Invitrogen, USA) following to manufacturer's information.

\section{Western blot analysis}

Cell lysate was prepared by using the RIPA buffer and the protein concentration was measured by BCA protein kit (Pierce, Rockford, IL). Total protein was isolated through $12 \%$ SDS-PAGE and transferred to the nitrocellulose membrane. Membrane was blocked with 5 $\%$ nonfat milk and incubated overnight with the primary antibodies (MMP-13 and GAPDH, Sigma). After washing in TBST, the membrane was incubated HRP-conjugated secondary antibodies. The membrane was detected with ECL (enhanced chemiluminescence).

\section{RNA extraction and $q R T-P C R$}

Total RNA was isolated by using the TRIzol reagent. Quantitative real-time PCR (qRT-PCR) was done by using SYBR GreenMix (Takara) on the MyiQ Real-Time PCR Detection System (BioRad, CA). All the primers were shown as following: MMP-13, 5'-TGCTTCCTGATGACGATGTAC-3', 5'-TCCTCGGAGACTGGTAATGG-3'; GAPDH, 5'-GACTCATGACCACAGTCCATGC-3', 5'-AGAGGCAGGGATGATGTTCTG-3'. Relative gene expression level was mesured by using the $\Delta \Delta \mathrm{CT}$ way and relative lncRNA expression was normalized to the U6 expression and mRNA expression level was normalized to GAPDH.

\section{Cell proliferation}

Cells were cultured in the 96-well plate and continued to culture for $0,24,48$ and 72 hours, respectively. Cell proliferation was measured by Cell Counting Kit 8 (CCK-8, Dojindo, Japan) following to the manufacturer's information. The end product was determined spectrophotometrically at the wavelength of $450 \mathrm{~nm}$.

\section{Luciferase reporter assay}

The amplified DNA sequences were cloned to the pmiR-RB-REPORT ${ }^{\mathrm{TM}}$ Vector to form MMP-13 3'UTR (WT) and mutated MMP-133'UTR (Mut) luciferase vectors. For the reporter assay, cells were plated in the 96-well plate and were co-transfected with miR-204$5 \mathrm{p}$ mimic and scramble and MMP-13 3'UTR (WT) or mutated MMP-133'UTR (Mut) luciferase vectors. After 48 hours, the luciferase activity was determined by using
Dual Luciferase Assay System (Promega, Madison, WI, USA) following to the manufacturer's information.

\section{Statistical analysis}

Value was shown as means \pm (standard deviation) SD. The statistical assay was performed by using SPSS 17.0 (IBM Corporation, USA). Student $t$ test was used to assess the significant difference between two groups and the differences between more than two groups were tested by one-way ANOVA. $P<0.05$ was thought to be statistically significant.

\section{ACKNOWLEDGMENTS AND FUNDING}

This work was supported by natural science foundation of shandong province (Grant Numbers: ZR2010HQ036 and ZR2014CQ042).

\section{CONFLICTS OF INTEREST}

None.

\section{REFERENCES}

1. Zhong Y, Huang Y, Santoso MB, Wu LD. Sclareol exerts anti-osteoarthritic activities in interleukin-1beta-induced rabbit chondrocytes and a rabbit osteoarthritis model. International journal of clinical and experimental pathology. 2015; 8:2365-2374.

2. Lim NH, Meinjohanns E, Meldal M, Bou-Gharios G, Nagase H. In vivo imaging of MMP-13 activity in the murine destabilised medial meniscus surgical model of osteoarthritis. Osteoarthritis and cartilage. 2014; 22:862-868.

3. Li W, Wu M, Jiang S, Ding W, Luo Q, Shi J. Expression of ADAMTs-5 and TIMP-3 in the condylar cartilage of rats induced by experimentally created osteoarthritis. Archives of oral biology. 2014; 59:524-529.

4. Haudenschild DR, Chen J, Pang N, Steklov N, Grogan SP, Lotz MK, D'Lima DD. Vimentin contributes to changes in chondrocyte stiffness in osteoarthritis. Journal of orthopaedic research. 2011; 29:20-25.

5. Goldring MB, Otero M. Inflammation in osteoarthritis. Current opinion in rheumatology. 2011; 23:471-478.

6. Goldring MB, Otero M, Plumb DA, Dragomir C, Favero M, El Hachem K, Hashimoto K, Roach HI, Olivotto E, Borzi RM, Marcu KB. Roles of inflammatory and anabolic cytokines in cartilage metabolism: signals and multiple effectors converge upon MMP-13 regulation in osteoarthritis. European cells \& materials. 2011; 21:202-220.

7. Pallu S, Francin PJ, Guillaume C, Gegout-Pottie P, Netter P, Mainard D, Terlain B, Presle N. Obesity affects the chondrocyte responsiveness to leptin in patients with osteoarthritis. Arthritis research \& therapy. 2010; 12:R112. 
8. Gruber HE, Ingram JA, Hoelscher GL, Zinchenko N, Hanley EN Jr, Sun Y. Asporin, a susceptibility gene in osteoarthritis, is expressed at higher levels in the more degenerate human intervertebral disc. Arthritis research \& therapy. 2009; 11:R47.

9. Iliopoulos D, Malizos KN, Tsezou A. Epigenetic regulation of leptin affects MMP-13 expression in osteoarthritic chondrocytes: possible molecular target for osteoarthritis therapeutic intervention. Annals of the rheumatic diseases. 2007; 66:1616-1621.

10. Colombini A, Cauci S, Lombardi G, Lanteri P, Croiset S, Brayda-Bruno M, Banfi G. Relationship between vitamin $\mathrm{D}$ receptor gene (VDR) polymorphisms, vitamin D status, osteoarthritis and intervertebral disc degeneration. The Journal of steroid biochemistry and molecular biology. 2013; 138C:24-40.

11. de Boer TN, van Spil WE, Huisman AM, Polak AA, Bijlsma JW, Lafeber FP, Mastbergen SC. Serum adipokines in osteoarthritis; comparison with controls and relationship with local parameters of synovial inflammation and cartilage damage. Osteoarthritis and cartilage. 2012.

12. Nie FQ, Sun M, Yang JS, Xie M, Xu TP, Xia R, Liu YW, Liu XH, Zhang EB, Lu KH, Shu YQ. Long noncoding RNA ANRIL promotes non-small cell lung cancer cell proliferation and inhibits apoptosis by silencing KLF2 and P21 expression. Molecular cancer therapeutics. 2015; 14:268-277.

13. Zhu H, Li X, Song Y, Zhang P, Xiao Y, Xing Y. Long noncoding RNA ANRIL is up-regulated in bladder cancer and regulates bladder cancer cell proliferation and apoptosis through the intrinsic pathway. Biochemical and biophysical research communications. 2015; 467:223-228.

14. Lin L, Gu ZT, Chen WH, Cao KJ. Increased expression of the long non-coding RNA ANRIL promotes lung cancer cell metastasis and correlates with poor prognosis. Diagnostic pathology. 2015; 10:14.

15. Sun L, Li Y, Yang B. Downregulated long non-coding RNA MEG3 in breast cancer regulates proliferation, migration and invasion by depending on p53's transcriptional activity. Biochemical and biophysical research communications. 2016; 478:323-329.

16. Yan H, Yuan J, Gao L, Rao J, Hu J. Long noncoding RNA MEG3 activation of p53 mediates ischemic neuronal death in stroke. Neuroscience. 2016; 337:191-199.

17. Liu X, Hou L, Huang W, Gao Y, Lv X, Tang J. The Mechanism of Long Non-coding RNA MEG3 for Neurons Apoptosis Caused by Hypoxia: Mediated by miR-181b12/15-LOX Signaling Pathway. Frontiers in cellular neuroscience. 2016; 10:201.

18. Li JQ, Hu SY, Wang ZY, Lin J, Jian S, Dong YC, Wu XF, Dai L, Cao LJ. Long non-coding RNA MEG3 inhibits microRNA-125a-5p expression and induces immune imbalance of Treg/Th17 in immune thrombocytopenic purpura. Biomedicine \& pharmacotherapy. 2016; 83:905911.
19. Gao Y, Lu X. Decreased expression of MEG3 contributes to retinoblastoma progression and affects retinoblastoma cell growth by regulating the activity of Wnt/beta-catenin pathway. Tumour biology. 2016; 37:1461-1469.

20. Kam Y, Rubinstein A, Naik S, Djavsarov I, Halle D, Ariel I, Gure AO, Stojadinovic A, Pan H, Tsivin V, Nissan A, Yavin E. Detection of a long non-coding RNA (CCAT1) in living cells and human adenocarcinoma of colon tissues using FIT-PNA molecular beacons. Cancer letters. 2014; 352:90-96.

21. Su S, Gao J, Wang T, Wang J, Li H, Wang Z. Long noncoding RNA BANCR regulates growth and metastasis and is associated with poor prognosis in retinoblastoma. Tumour biology. 2015; 36:7205-7211.

22. McCleland ML, Mesh K, Lorenzana E, Chopra VS, Segal E, Watanabe C, Haley B, Mayba O, Yaylaoglu M, Gnad F, Firestein R. CCAT1 is an enhancer-templated RNA that predicts BET sensitivity in colorectal cancer. The Journal of clinical investigation. 2016.

23. Li PF, Chen SC, Xia T, Jiang XM, Shao YF, Xiao BX, Guo JM. Non-coding RNAs and gastric cancer. World journal of gastroenterology. 2014; 20:5411-5419.

24. He X, Tan X, Wang X, Jin H, Liu L, Ma L, Yu H, Fan Z. C-Myc-activated long noncoding RNA CCAT1 promotes colon cancer cell proliferation and invasion. Tumour biology. 2014; 35:12181-12188.

25. Guo Q, Qian Z, Yan D, Li L, Huang L. LncRNA-MEG3 inhibits cell proliferation of endometrial carcinoma by repressing Notch signaling. Biomedicine \& pharmacotherapy. 2016; 82:589-594.

26. Su W, Xie W, Shang Q, Su B. The Long Noncoding RNA MEG3 Is Downregulated and Inversely Associated with VEGF Levels in Osteoarthritis. BioMed research international. 2015; 2015:356893.

27. Wang XS, Zhang Z, Wang HC, Cai JL, Xu QW, Li MQ, Chen YC, Qian XP, Lu TJ, Yu LZ, Zhang Y, Xin DQ, Na YQ, Chen WF. Rapid identification of UCA1 as a very sensitive and specific unique marker for human bladder carcinoma. Clinical cancer research. 2006; 12:4851-4858.

28. Pan J, Li X, Wu W, Xue M, Hou H, Zhai W, Chen W. Long non-coding RNA UCA1 promotes cisplatin/gemcitabine resistance through CREB modulating miR-196a-5p in bladder cancer cells. Cancer letters. 2016; 382:64-76.

29. He A, Hu R, Chen Z, Liao X, Li J, Wang D, Lv Z, Liu Y, Wang F, Mei H. Role of long noncoding RNA UCA1 as a common molecular marker for lymph node metastasis and prognosis in various cancers: a meta-analysis. Oncotarget. 2016; 8:19371943. https://doi.org/10.18632/oncotarget.12463.

30. Li X, Wu Y, Liu A, Tang X. Long non-coding RNA UCA1 enhances tamoxifen resistance in breast cancer cells through a miR-18a-HIF1alpha feedback regulatory loop. Tumour biology. 2016; 37:14733-14743.

31. Yang YT, Wang YF, Lai JY, Shen SY, Wang F, Kong J, Zhang W, Yang HY. Long non-coding RNA UCA1 
contributes to the progression of oral squamous cell carcinoma by regulating the WNT/beta-catenin signaling pathway. Cancer science. 2016; 107:1581-1589.

32. Lee JJ, Kim M, Kim HP. Epigenetic regulation of long noncoding RNA UCA1 by SATB1 in breast cancer. BMB reports. 2016; 49:578-583.

33. Wu C, Luo J. Long Non-Coding RNA (lncRNA) Urothelial Carcinoma-Associated 1 (UCA1) Enhances Tamoxifen Resistance in Breast Cancer Cells via Inhibiting mTOR Signaling Pathway. Medical science monitor. 2016; 22:3860-3867.

34. Fang Q, Chen X, Zhi X. Long Non-Coding RNA (LncRNA) Urothelial Carcinoma Associated 1 (UCA1) Increases Multi-Drug Resistance of Gastric Cancer via Downregulating miR-27b. Medical science monitor. 2016; 22:3506-3513.

35. Li W, Xie P, Ruan WH. Overexpression of lncRNA UCA1 promotes osteosarcoma progression and correlates with poor prognosis. Journal of bone oncology. 2016; 5:80-85.

36. Li Y, Wang T, Chen D, Yu Z, Jin L, Ni L, Yang S, Mao X, Gui Y, Lai Y. Identification of long-non coding RNA UCA1 as an oncogene in renal cell carcinoma. Molecular medicine reports. 2016; 13:3326-3334.
37. Tao K, Yang J, Hu Y, Sun Y, Tan Z, Duan J, Zhang F, Yan $\mathrm{H}$, Deng A. Clinical significance of urothelial carcinoma associated 1 in colon cancer. International journal of clinical and experimental medicine. 2015; 8:21854-21860.

38. Wang F, Ying HQ, He BS, Pan YQ, Deng QW, Sun HL, Chen J, Liu X, Wang SK. Upregulated IncRNA-UCA1 contributes to progression of hepatocellular carcinoma through inhibition of miR-216b and activation of FGFR1/ ERK signaling pathway. Oncotarget. 2015; 6:7899-7917. https://doi.org/10.18632/oncotarget.3219.

39. Jiao C, Song Z, Chen J, Zhong J, Cai W, Tian S, Chen S, Yi Y, Xiao Y. lncRNA-UCA1 enhances cell proliferation through functioning as a ceRNA of Sox 4 in esophageal cancer. Oncology reports. 2016; 36:2960-2966.

40. Chen P, Wan D, Zheng D, Zheng Q, Wu F, Zhi Q. Long non-coding RNA UCA1 promotes the tumorigenesis in pancreatic cancer. Biomedicine \& pharmacotherapy. 2016; 83:1220-1226.

41. Lu L, Shen Y, Tseng KF, Liu W, Duan H, Meng W. Silencing of UCA1, a poor prognostic factor, inhibited the migration of endometrial cancer cell. Cancer biomarkers: section A of Disease markers. 2016; 17:171-177. 